\title{
Taxas respiratórias de sementes de Eugenia pyriformis Cambess. dispersas em diferentes estádios de maturação
}

\author{
(1D) Edmir Vicente Lamarca ${ }^{1,4}$, iD João José Dias Parisi²e iD Claudio José Barbedoº
}

Recebido: 11.11.2019; aceito: 02.06.2020

Como citar: Lamarca, E.V., Parisi, J.J.D. \& Barbedo, C.J. 2020. Taxas respiratórias de sementes de Eugenia pyriformis Cambess. dispersas em diferentes estádios de maturação. Hoehnea 47: e1012019. http://dx.doi.org/10.1590/2236-8906-101/2019.

\begin{abstract}
Respiratory rates of Eugenia pyriformis Cambess. seeds of different maturity at shedding). In the present study, the respiratory rates of Eugenia pyriformis Cambess. seeds were analyzed according to the material origin, submitted to different drying levels and incubation temperatures, including the analysis of environmental conditions during seed formation. The results showed variations in the respiratory intensity of these seeds as a function of origin, mainly among freshly dispersed seeds (without drying) or when submitted to more severe drying and adverse germination temperatures. The highest the seed water content at shedding, the highest the respiration rate. Such variations suggest differences in the maturity of seeds from different regions, with influences of environmental conditions during seed formation.
\end{abstract}

Keywords: Myrtaceae, recalcitrance, seed conservation

RESUMO - (Taxas respiratórias de sementes de Eugenia pyriformis Cambess. dispersas em diferentes estádios de maturação). No presente estudo, as taxas respiratórias das sementes de Eugenia pyriformis Cambess. foram analisadas de acordo com a origem do material, submetidas a diferentes níveis de secagem e temperaturas de incubação, incluindo a análise das condições ambientais durante a formação das sementes. Os resultados mostraram variações na intensidade respiratória das sementes em função da origem, principalmente entre sementes recém-dispersas (sem secagem) ou quando submetidas a secagens mais severas e temperaturas desfavoráveis à germinação. Quanto maior o teor de água das sementes na dispersão, maior a taxa de respiração. Tais variações sugerem diferenças na maturidade de sementes de diferentes regiões, com influências das condições ambientais durante a formação das sementes.

Palavras-chave: conservação de sementes, Myrtaceae, recalcitrância

\section{Introdução}

Eugenia pyriformis Cambess. pertence à família Myrtaceae e é conhecida popularmente como uvaieira ou uvaia, sendo uma espécie nativa do Brasil, de ocorrência em florestas tropicais e subtropicais. Suas sementes são sensíveis à dessecação e normalmente dispersas com elevado teor de água, o que dificulta seu armazenamento, pois mantém elevado metabolismo e, consequentemente, rápida deterioração (Andrade \& Ferreira 2000, Delgado \&
Barbedo 2007, Scalon et al. 2012). Desta forma, devido as suas características fisiológicas, sementes de Eugenia pyriformis apresentam-se como um interessante modelo para maior compreensão de processos que envolvam a sensibilidade à dessecação.

Na década de 1970, as sementes foram classificadas quanto a sua tolerância à dessecação e quanto ao seu período de armazenamento (Roberts 1973). Aquelas sementes denominadas como ortodoxas são tolerantes à dessecação e ao armazenamento. Já aquelas sementes denominadas como

1. Universidade Ibirapuera, Avenida Interlagos, Chácara Flora, 1329, 04661-100 São Paulo, SP, Brasil

2. Instituto Florestal, Estação Experimental de Tupi, Rodovia Luiz de Queiroz, Km 149, 13428-000 Piracicaba, SP, Brasil

3. Instituto de Botânica, Núcleo de Pesquisa em Sementes, Avenida Miguel Stéfano, 3687, 04301-012 São Paulo, SP, Brasil

4. Autor para correspondência: edmirvicente18@gmail.com 
recalcitrantes são sensíveis à dessecação ao armazenamento (Roberts 1973, Walters 2000, Berjak \& Pammenter 2007, Silva et al. 2018, Mayrinck et al. 2019, Stavrinides et al. 2019).

Recentemente, tem-se considerado que as sementes sensíveis à dessecação, ou seja, as recalcitrantes podem ser sementes dispersas sem ter completado seu processo de maturação. Nesse caso, quanto mais distante a semente se encontra do ponto de maturidade fisiológica no momento de sua dispersão, mais sensível à dessecação será (Barbedo et al. 2013). Com isso, as sementes recalcitrantes não passando pela fase de secagem da maturação, característica das sementes tolerantes à dessecação, ou seja, as ortodoxas, não podem ser conservadas por períodos superiores a semanas ou alguns meses, uma vez que a secagem é a principal forma de se reduzir o metabolismo tanto da semente quanto de agentes causadores de danos, como micro-organismos e insetos (Silva et al. 2018, Mayrinck et al. 2019, Stavrinides et al. 2019).

A conservação da viabilidade das sementes recalcitrantes em armazenamento é, atualmente, um dos maiores desafios da ciência das sementes. Contudo, pouco ainda se conhece sobre a fisiologia dessas sementes quanto à sua rápida deterioração. Alguns estudos sugerem que a elevada atividade respiratória, o metabolismo desordenado e a baixa proteção às espécies reativas de oxigênio nos sistemas de membranas estão entre os principais eventos que envolvem a rápida deterioração de sementes sensíveis à dessecação, dificultando o seu armazenamento (Leprince et al. 1999, Berjak \& Pammenter 2007).

A sensibilidade à dessecação em sementes recalcitrantes pode estar associada à incapacidade da diminuição do metabolismo durante a secagem, com possíveis aumentos de danos peroxidativos, como visto para sementes de Castanea sativa, as quais são classificadas como recalcitrantes (Leprince et al. 1999). Outras sementes recalcitrantes podem, inclusive, iniciar a germinação antes mesmo de sua dispersão, como ocorre em Inga vera, aumentando sua sensibilidade à dessecação e acelerando ainda mais o metabolismo (Caccere et al. 2013, Bonjovani \& Barbedo 2019), comportamentos que dificultam o armazenamento. Nas sementes tolerantes à dessecação, por sua vez, é evidente a mudança no perfil metabólico na fase que antecede a secagem da maturação, quando há um desligamento das atividades, especialmente as taxas respiratórias (Hell et al. 2019).

Diversas características físicas e fisiológicas das sementes podem variar de acordo com a origem do material, devido à variabilidade genética, respostas adaptativas ou em decorrência às condições ambientais, apresentado variações no armazenamento e nos limites e exigências térmicas para a germinação (Daws et al. 2004, Mattana et al. 2012). Variações na temperatura do ar durante a formação das sementes (representada pelo conceito de graus-dia) podem, inclusive, influenciar a maturidade fisiológica, afetando o teor de água inicial, a germinação, os níveis de dormência e a sensibilidade à dessecação (Daws et al. 2006). Contudo, além dessas características, a atividade metabólica após a dispersão também pode diferir em função do padrão de desenvolvimento das sementes (Wilson et al. 1970, Berjak \& Pammenter 2007).

Todavia, na literatura científica, poucos são os estudos que avaliam a respiração de sementes, principalmente, as sensíveis à dessecação, sejam estas denominadas também recalcitrantes ou ortodoxas imaturas. Assim, vislumbrando maior compreensão sobre o comportamento dessas sementes, indagam-se aqui alguns questionamentos. Existem variações na intensidade respiratória, sendo estas possíveis de serem diagnosticadas, para sementes procedentes de diferentes regiões e condições ambientais durante a formação. Ainda, tais variações estão associadas a diferentes estágios de maturação e níveis de sensibilidade à dessecação. Sob tal contexto, sabendo da importância da respiração para a deterioração e armazenamento e da dificuldade de armazenamento de sementes sensíveis à dessecação, além de poucas informações na literatura científica sobre essas questões, se faz por de grande importância, estudos que analisem as taxas respiratórias de sementes sensíveis à dessecação, visando trazer substancial contribuição para a compreensão de processos que envolvem sua rápida deterioração e subsídios para adoção de procedimentos e estratégias que contribuam para a conservação da biodiversidade.

Desta forma, tendo como hipótese que as taxas de deterioração de sementes recalcitrantes estão relacionadas com suas taxas respiratórias, e que estas são decorrentes do grau de maturação das sementes, da temperatura de armazenamento e do seu teor de água, o presente estudo teve como objetivo analisar as taxas respiratórias de sementes de Eugenia pyriformis Cambess. em função da origem do material, submetidas a diferentes níveis de secagem e diferentes temperaturas de incubação, incluindo a análise das condições ambientais durante a formação das sementes.

\section{Material e Métodos}

Origem do material - as sementes de Eugenia pyriformis Cambess. foram obtidas de frutos maduros recém-dispersos (Lamarca et al. 2013). Os frutos foram procedentes do ano de 2010 de sete diferentes regiões, conforme apresentado em detalhes na tabela 1. Adotaram-se siglas, de acordo com a região de coleta, da seguinte forma: Lavras (LAV), Campinas (CAM), São Bernardo do Campo (SBC), São Bento do Sapucaí (SBS), Jumirim (JUM), Ribeirão Preto (RIB) e Itaberá (ITA). Após a coleta, as sementes foram extraídas dos frutos, com auxílio de peneira e água corrente, e armazenadas em câmara fria a $7^{\circ} \mathrm{C}$ até o início dos experimentos, não excedendo sete dias (Andrade \& Ferreira 2000, Delgado $\&$ Barbedo 2007). 


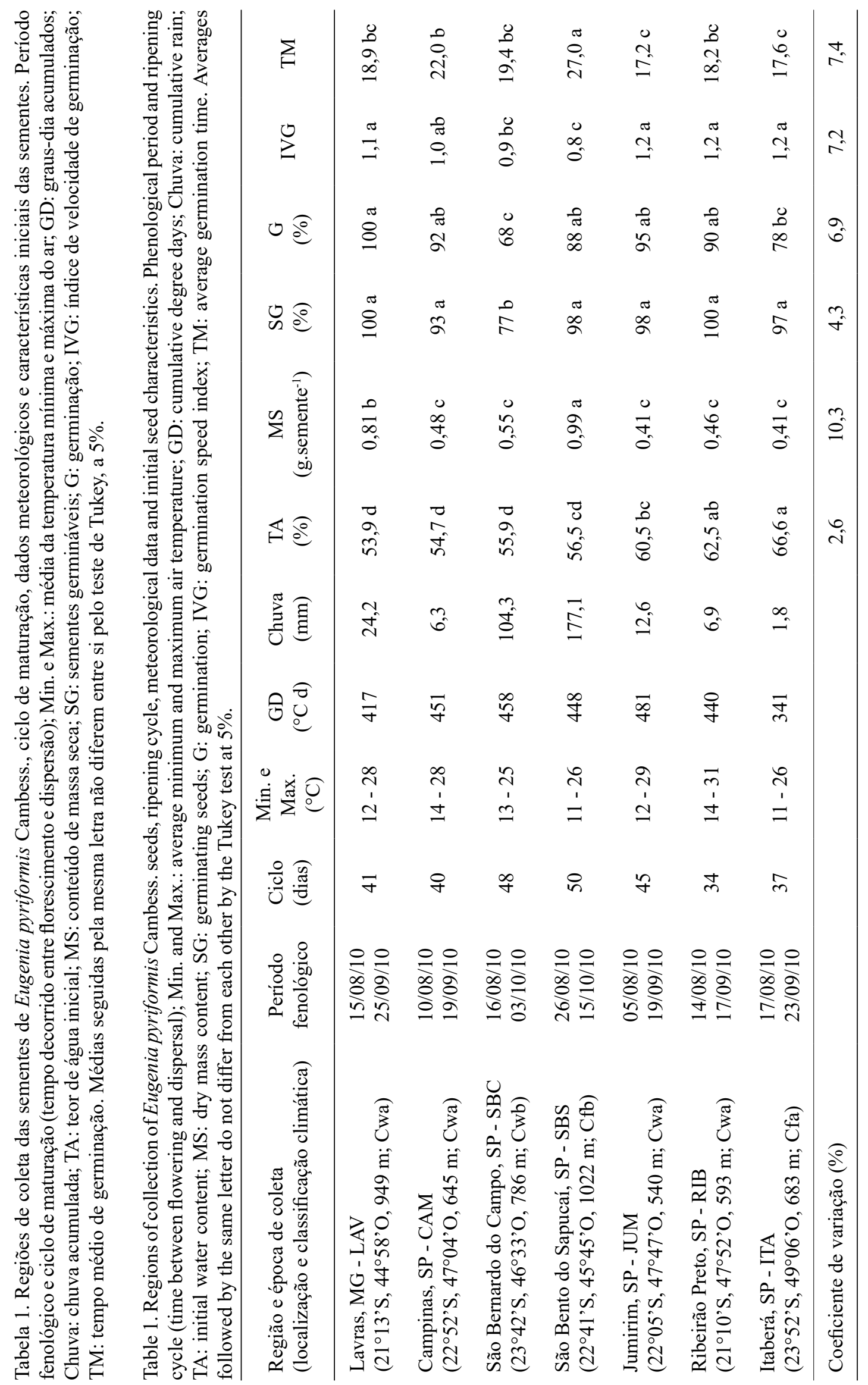


Dados de estações meteorológicas - foram obtidos de estações localizadas próximas às áreas de coleta dados diários de precipitação pluvial (mm) e temperatura (mínima e máxima) do $\operatorname{ar}\left({ }^{\circ} \mathrm{C}\right)$. Inicialmente inflorescências das árvores foram marcadas no período de máximo florescimento da espécie e as sementes coletadas imediatamente após a dispersão natural (até 24 horas da dispersão). Do período compreendido entre o florescimento e a dispersão foram calculados os graus-dia acumulados $\left({ }^{\circ} \mathrm{C}\right.$ dias), segundo as equações propostas por Villa Nova et al. (1972), considerando-se a temperatura base de $10{ }^{\circ} \mathrm{C}$ (Pedro Junior et al. 1977). Foi calculado o balanço hídrico sequencial, segundo o modelo proposto por Thornthwaite \& Mather (1955), em nível de escala decendial, com capacidade de água disponível (CAD) de $125 \mathrm{~mm}$ (Rolim et al. 2007). Calcularam-se também os valores médios das temperaturas máxima e mínima do ar e da chuva acumulada ( $\mathrm{mm}$ ).

Caracterização inicial - após a coleta, as sementes foram caracterizadas inicialmente quanto ao teor de água, conteúdo de massa seca e germinação. $O$ teor de água e o conteúdo de massa seca foram determinados gravimetricamente pelo método de estufa a $103{ }^{\circ} \mathrm{C}$ por 17 horas, sendo os resultados apresentados em \% de base úmida para o teor de água e em g.semente ${ }^{-1}$ para o conteúdo de massa seca (Brasil 2009).

$\mathrm{O}$ teste de germinação foi conduzido em sala de germinação de $25 \mathrm{~m}^{2}$, com temperatura continua de $25 \pm$ $1{ }^{\circ} \mathrm{C}$, com luz constante e com umidade relativa de $80 \% \pm$ $10 \%$. A temperatura da sala era garantida por condicionador de ar e iluminada por oito lâmpadas fluorescentes de $40 \mathrm{~W}$ cada. Para o teste de germinação foi utilizado o método rolo de papel (Brasil 2009). As avaliações foram realizadas a cada 3 dias, durante 70 dias (Delgado \& Barbedo 2007, Lamarca et al. 2011), registrando-se as sementes que emitiram raiz primária, para o cálculo de sementes germináveis e as que produziram plântulas normais, para o cálculo de germinação, ambos os resultados apresentados em porcentagem. Foi calculado, também, o índice de velocidade de germinação (IVG), utilizando a fórmula de Maguire (1962) e o tempo médio de germinação, segundo Borghetti \& Ferreira (2004).

Avaliação da taxa respiratória - a respiração das sementes de Eugenia pyriformis foi determinada por meio de analisador de oxigênio $\left(\mathrm{O}_{2}\right)$ e dióxido de carbono $\left(\mathrm{CO}_{2}\right)$, equipamento "Illinois Instruments, Inc., Johnsburg, EUA (modelo 6600)", segundo metodologia descrita por Lamarca \& Barbedo (2012). Para tanto, amostras de sementes foram incubadas na ausência de luz em embalagens herméticas de $600 \mathrm{~mL}$. Antes da incubação foi determinada a massa fresca (g) e o teor de água (conforme descrito anteriormente). Foi calculado também o volume total do ar das embalagens. Esses dados foram utilizados depois na conversão dos valores em porcentagem, obtidos pelo equipamento, para micromol $(\mu \mathrm{mol})$ de $\mathrm{O}_{2}$ ou $\mathrm{CO}_{2}$ por grama de massa seca por dia (Lamarca \& Barbedo 2012).

Com base em experimentos realizados de forma prévia, da média de consumo de $\mathrm{O}_{2}$ e produção de $\mathrm{CO}_{2}$ pelas sementes de Eugenia pyriformis, as avaliações da respiração foram realizadas no intervalo de um dia. Os valores obtidos nas avaliações foram somados e divididos pela massa seca total da amostra e pelo número de dias de incubação (total de 10 dias), obtendo-se, assim, valores médios de $\mathrm{O}_{2}$ ou $\mathrm{CO}_{2}$ expressos em micromol por grama de massa seca por dia $\left(\mu \mathrm{mol} \mathrm{O}_{2}\right.$ ou $\mathrm{CO}_{2} \mathrm{~g} \mathrm{MS}^{-1} \mathrm{~d}^{-1}$ ) (Lamarca \& Barbedo 2012). Calculou-se também o quociente respiratório $(\mathrm{QR})$, ou seja, a relação entre a produção de $\mathrm{CO}_{2}$ e o consumo de $\mathrm{O}_{2}(\mathrm{QR}=$ $\mathrm{CO}_{2} \mathrm{O}_{2}^{-1}$ ), segundo a equação descrita por Kader \& Saltveit (2002). Ambas as variáveis $\left(\mathrm{CO}_{2}\right.$ e $\left.\mathrm{O}_{2}\right) \mathrm{em} \mu \mathrm{mol} \mathrm{g} \mathrm{MS}^{-1} \mathrm{~d}^{-1}$.

Taxas respiratórias de sementes com diferentes níveis de secagem - amostras de sementes das diferentes regiões foram submetidas à secagem controlada e avaliadas quanto à respiração. Para tanto, após a retirada da amostra controle (sem secagem), as sementes foram submetidas a dois níveis de secagem controlada em estufa, com circulação forçada de ar. As sementes foram dispostas em prateleiras forradas com tela de polietileno, em camadas simples sem sobreposição. A secagem foi realizada de forma intermitente, com 10 horas a $40{ }^{\circ} \mathrm{C}$, seguidas de 14 horas de repouso a $20-25^{\circ} \mathrm{C}$ (Delgado \& Barbedo 2007). Periodicamente, com base no valor da massa seca das sementes, amostras foram retiradas e avaliadas quanto ao teor de água. Este procedimento foi repetido até que as sementes atingissem dois teores de água pré-estabelecidos de $50 \%$ e $40 \%$ de base úmida, ou seja, quando $50 \%$ do vigor e da germinação, respectivamente, são perdidos (Delgado \& Barbedo 2007). Desta forma, constituíram-se diferentes níveis de secagem, ou seja, sementes sem secagem $\left(\mathrm{S}_{0}\right)$ e sementes secas até o primeiro $\left(\mathrm{S}_{\mathrm{I}}\right)$ e segundo $\left(\mathrm{S}_{\mathrm{II}}\right)$ nível. Após atingidos os desejados níveis de secagem, as sementes foram avaliadas quanto a taxa respiratória, conforme descrito anteriormente.

Taxas respiratórias em diferentes temperaturas de incubação - avaliação da respiração foi realizada conforme descrição anterior. Para tal experimento, amostras de sementes recémdispersas, ou seja, sem secagem, das diferentes regiões de coleta foram acondicionadas em frascos herméticos e estes incubados em câmaras de incubação do tipo B.O.D., modelo 347 CD (Fanem, São Paulo), as quais estavam ajustas nas temperaturas de $10,15,20,25,30$ e $35^{\circ} \mathrm{C}$, com ausência de luz.

Delineamento experimental e análise estatística - o delineamento experimental foi o inteiramente casualizado em esquema fatorial, associando-se regiões de coleta (origem do material) e níveis de secagem ( 7 × 3 ) e regiões de coleta (origem do material) e temperaturas de incubação $(7 \times 6)$, com três repetições de 20 sementes. Nos resultados da caracterização inicial compararam-se as regiões de coleta (três repetições de 20 sementes). Os dados obtidos foram submetidos à análise de variância (teste F), ao nível de $5 \%$ de significância. Quando pertinente, as médias foram comparadas entre si pelo teste de Tukey, também ao nível de 5\% (Santana \& Ranal 2004). 


\section{Resultados e Discussão}

A análise de variância para os dados de consumo de oxigênio $\left(\mathrm{O}_{2}\right)$ e produção de dióxido de carbono $\left(\mathrm{CO}_{2}\right)$ apresentou interação significativa entre os fatores regiões de coleta e níveis de secagem. Já para quociente respiratório não houve interação significativa e nem diferenças entre os níveis dentro dos fatores regiões de coleta e secagem. Portanto, as taxas respiratórias podem ser analisadas tanto pelo consumo de oxigênio quanto pela produção de dióxido de carbono, uma vez que não houve evidente fermentação, tampouco oxidação não respiratória (Labouriau 1983, Kader \& Saltveit 2002), como ocorre, por exemplo, em sementes de Caesalpinia echinata Lam. (Lamarca \& Barbedo 2012).

As taxas respiratórias de sementes de Eugenia pyriformis logo após a dispersão (sem secagem) variaram conforme a sua procedência, sendo maiores para RIB e ITA e menores para LAV, CAM e SBS (tabela 2). Curiosamente, as primeiras estão entre as que apresentaram maior teor de água inicial que, segundo Barbedo et al. (2013), as situariam como as dispersas mais imaturas que as demais. LAV e CAM, por sua vez, estariam entre as mais maduras uma vez que seu teor de água na dispersão esteve entre os mais baixos.

O primeiro nível de secagem resultou em respostas diferentes conforme a origem das sementes. Para as que apresentavam teor inicial de água mais baixo a secagem praticamente não alterou as taxas respiratórias; contudo, para as de teor inicial mais alto a secagem resultou em redução nas taxas respiratórias. Tal resultado foi hipotetizado por Barbedo et al. (2013) que afirmaram que simulações artificiais dos processos pelos quais a semente passa durante a maturação, como pequenas reduções na disponibilidade hídrica, podem promover pequenos ganhos, semelhantes ao amadurecimento natural. Todavia, déficits hídricos maiores poderiam causar alterações indesejadas, como os verificados no segundo nível de secagem, que aumentou as taxas respiratórias nessas sementes (tabela 2). Esse aumento na respiração pode indicar, inclusive, uma condição de deterioração, quando a semente respira mais intensamente, como observado em Caesalpinia echinata (Lamarca \& Barbedo 2012).

Outros estudos envolvendo sementes sensíveis à dessecação verificaram que as taxas respiratórias diminuem quando as sementes são secas a teores de água que afetam a germinação ou a integridade das membranas, em conjunção com o aumento da viscosidade do citoplasma, formações de espécies reativas de oxigênio e metabolismo desordenado em tecidos estressados, como observado por Leprince et al. (1999), que estudaram a respiração em relação à secagem de sementes de Castanea sativa. O estudo de respiração realizado com sementes de Eugenia dysenterica, também demonstrou variações nas taxas respiratórias após a secagem. Sementes dessa espécie foram submetidas à secagem em sílica gel e em estufa em períodos de 12, 24, 48 e 72 horas. A secagem em sílica gel proporcionou aumento linear na respiração das sementes. Já a secagem em estufa proporcionou um aumento da respiração nas primeiras horas de secagem, mas depois houve um decréscimo da respiração. Ainda neste estudo, as análises de germinação e vigor acompanharam a segunda resposta respiratória (Silva et al. 2017).

Em relação à temperatura, a análise de variância apresentou interação significativa entre os fatores regiões de coleta e temperaturas de incubação, para as variáveis consumo de oxigênio, produção de dióxido de carbono e quociente respiratório (tabela 3 ). Em geral, nota-se que o consumo de oxigênio e a produção de dióxido de carbono aumentam à medida que aumenta a temperatura de incubação. Curiosamente, porém, mesmo se reduzindo a temperatura para valores próximos à refrigeração comum de conservação de sementes e alimentos (inferior a $10^{\circ} \mathrm{C}$, Barbedo et al. 2002), ainda há intensa atividade metabólica nas sementes de Eugenia pyriformis, vista pelas taxas respiratórias. Portanto, a conservação dessas sementes em ambiente refrigerado não é suficiente para seu armazenamento em longo prazo, uma vez que há, ainda, intensa atividade metabólica sob essa condição.

Embora as respostas respiratórias sejam semelhantes nas diferentes procedências, observam-se algumas diferenças importantes. Por exemplo, as sementes oriundas de RIB, com mais elevado teor de água na dispersão, incubadas a 10 ou a $15^{\circ} \mathrm{C}$ já consumiam oxigênio e produziam dióxido de carbono em quantidades equivalentes as sementes de LAV, com menor teor de água na dispersão, a 30 ou a $35^{\circ} \mathrm{C}$ (tabela 3). As diferenças entre as intensidades respiratórias também ocorreram para as sementes das regiões de ITA, CAM e SBS: ITA a $20^{\circ} \mathrm{C}$ apresentou valores semelhantes de consumo de oxigênio e produção de dióxido de carbono a CAM e SBS, quando estas estavam, respectivamente, a 25 e $30{ }^{\circ} \mathrm{C}$ (tabela 3). Como dito anteriormente, essas diferenças na intensidade respiratória podem estar relacionadas com o teor de água inicial (sementes sem secagem) e a maturidade das sementes (tabela 1).

$\mathrm{O}$ quociente respiratório também modificou em função da temperatura. Verificou-se que a relação entre a produção de dióxido de carbono e consumo de oxigênio tendeu a aproximar-se de 1 na faixa térmica de 20 a $30{ }^{\circ} \mathrm{C}$ (tabela 3), curiosamente a faixa adequada para a germinação e desenvolvimento de sementes de Eugenia pyriformis (Lamarca et al. 2011). Já fora dessa faixa térmica adequada, ou seja, temperaturas nas quais a germinação e o desenvolvimento de plântulas normais diminuem, por exemplo, a 10 ou a $15{ }^{\circ} \mathrm{C}$ verifica-se, na maioria das situações, um quociente respiratório acima de 1 (tabela 3 ). Outro interessante aspecto foi que as sementes de RIB apresentaram quociente respiratório abaixo de 1 em todas as temperaturas de incubação, sugerindo possíveis reações oxidativas além da respiração (tabela 3 ).

Cabe enfatizar que na incubação de sementes sob baixas temperaturas, pode haver menor difusão de oxigênio, 
Tabela 2. Consumo de oxigênio, produção de dióxido de carbono e quociente respiratório de sementes de Eugenia pyriformis Cambess. de distintas regiões de coleta, submetidas a diferentes níveis de secagem. Médias seguidas pela mesma letra (minúscula compara regiões de coleta e maiúscula compara níveis de secagem) não diferem entre si pelo teste de Tukey, a 5\%. $\mathrm{S}_{0}$ : sem secagem; $\mathrm{S}_{\mathrm{I}}$ : primeiro nível de secagem; $\mathrm{S}_{\mathrm{II}}$ : segundo nível de secagem. LAV: Lavras; CAM: Campinas; SBC: São Bernardo do Campo; SBS: São Bento do Sapucaí; JUM: Jumirim; RIB: Ribeirão Preto; ITA: Itaberá.

Table 2. Oxygen consumption, carbon dioxide production and respiratory quotient of Eugenia pyriformis Cambess. seeds from different collection regions, submitted to different drying levels. Means followed by the same letter (lower case compare collection regions and upper case compare drying levels) do not differ from each other by the Tukey test at $5 \%$. $\mathrm{S}_{0}$ : without drying; $\mathrm{S}_{\mathrm{I}}$ : first level of drying; $\mathrm{S}_{\mathrm{II}}$ : second level of drying. LAV: Lavras; CAM: Campinas; SBC: São Bernardo do Campo; SBS: São Bento do Sapucaí; JUM: Jumirim; RIB: Ribeirão Preto; ITA: Itaberá.

\begin{tabular}{|c|c|c|c|c|c|}
\hline \multirow{2}{*}{$\begin{array}{l}\text { Região de coleta } \\
\text { (Teor de água nos níveis de secagem, \%) }\end{array}$} & \multicolumn{5}{|c|}{ Nível de secagem (\%) } \\
\hline & $\mathrm{S}_{0}$ & & $\mathrm{~S}_{\mathrm{I}}$ & & $\mathrm{S}_{\mathrm{II}}$ \\
\hline \multicolumn{6}{|c|}{ Oxigênio $\left(\mu \mathrm{mol} \mathrm{O}_{2} \mathrm{gMS}^{-1} \mathrm{~d}^{-1}\right)$} \\
\hline $\operatorname{LAV}\left(\mathrm{S}_{0}=54 ; \mathrm{S}_{\mathrm{I}}=50 ; \mathrm{S}_{\mathrm{II}}=40\right)$ & $31,7 \mathrm{dA}$ & & 36,9 aA & & $38,0 \mathrm{cdA}$ \\
\hline $\operatorname{CAM}\left(\mathrm{S}_{0}=55 ; \mathrm{S}_{\mathrm{I}}=49 ; \mathrm{S}_{\mathrm{II}}=39\right)$ & $45,5 \mathrm{cA}$ & & $41,9 \mathrm{aA}$ & & $49,9 \mathrm{cA}$ \\
\hline $\operatorname{SBC}\left(\mathrm{S}_{0}=56 ; \mathrm{S}_{\mathrm{I}}=51 ; \mathrm{S}_{\mathrm{II}}=39\right)$ & $45,7 \mathrm{bcA}$ & & $39,5 \mathrm{aA}$ & & $46,9 \mathrm{cA}$ \\
\hline $\operatorname{SBS}\left(\mathrm{S}_{0}=57 ; \mathrm{S}_{\mathrm{I}}=51 ; \mathrm{S}_{\mathrm{II}}=39\right)$ & $42,7 \mathrm{cdA}$ & & $43,4 \mathrm{aA}$ & & $32,2 \mathrm{dA}$ \\
\hline $\operatorname{JUM}\left(\mathrm{S}_{0}=61 ; \mathrm{S}_{\mathrm{I}}=50 ; \mathrm{S}_{\mathrm{II}}=41\right)$ & $48,2 \mathrm{bcA}$ & & $36,4 \mathrm{aB}$ & & $25,9 \mathrm{dC}$ \\
\hline $\operatorname{RIB}\left(\mathrm{S}_{0}=63 ; \mathrm{S}_{\mathrm{I}}=50 ; \mathrm{S}_{\mathrm{II}}=41\right)$ & $67,2 \mathrm{aB}$ & & $44,8 \mathrm{aC}$ & & 83,1 aA \\
\hline $\operatorname{ITA}\left(\mathrm{S}_{0}=67 ; \mathrm{S}_{\mathrm{I}}=48 ; \mathrm{S}_{\mathrm{II}}=41\right)$ & $58,6 \mathrm{abAl}$ & & $48,5 \mathrm{aB}$ & & $63,3 \mathrm{bA}$ \\
\hline \multicolumn{6}{|l|}{ Coeficiente de Variação (\%) 11,1 } \\
\hline \multicolumn{6}{|c|}{ Dióxido de Carbono $\left(\mu \mathrm{mol} \mathrm{CO} \mathrm{gMS}^{-1} \mathrm{~d}^{-1}\right)$} \\
\hline $\operatorname{LAV}\left(\mathrm{S}_{0}=54 ; \mathrm{S}_{\mathrm{I}}=50 ; \mathrm{S}_{\mathrm{II}}=40\right)$ & $32,4 \mathrm{cA}$ & & $35,1 \mathrm{aA}$ & & $37,7 \mathrm{bcA}$ \\
\hline $\operatorname{CAM}\left(\mathrm{S}_{0}=55 ; \mathrm{S}_{\mathrm{I}}=49 ; \mathrm{S}_{\mathrm{II}}=39\right)$ & $41,5 \mathrm{cA}$ & & $38,3 \mathrm{aA}$ & & $43,9 \mathrm{bA}$ \\
\hline $\mathrm{SBC}\left(\mathrm{S}_{0}=56 ; \mathrm{S}_{\mathrm{I}}=51 ; \mathrm{S}_{\mathrm{II}}=39\right)$ & $44,7 \mathrm{bcA}$ & & 39,1 aA & & $45,9 \mathrm{bA}$ \\
\hline $\operatorname{SBS}\left(\mathrm{S}_{0}=57 ; \mathrm{S}_{\mathrm{I}}=51 ; \mathrm{S}_{\mathrm{II}}=39\right)$ & $40,2 \mathrm{cA}$ & & $39,1 \mathrm{aA}$ & & $35,9 \mathrm{bcA}$ \\
\hline $\operatorname{JUM}\left(\mathrm{S}_{0}=61 ; \mathrm{S}_{\mathrm{I}}=50 ; \mathrm{S}_{\mathrm{II}}=41\right)$ & $45,8 \mathrm{bcA}$ & & $33,3 \mathrm{aB}$ & & $25,3 \mathrm{cB}$ \\
\hline $\operatorname{RIB}\left(\mathrm{S}_{0}=63 ; \mathrm{S}_{\mathrm{I}}=50 ; \mathrm{S}_{\mathrm{II}}=41\right)$ & $69,1 \mathrm{aA}$ & & $38,6 \mathrm{aB}$ & & $72,5 \mathrm{aA}$ \\
\hline $\operatorname{ITA}\left(\mathrm{S}_{0}=67 ; \mathrm{S}_{\mathrm{I}}=48 ; \mathrm{S}_{\mathrm{II}}=41\right)$ & $57,5 \mathrm{abAl}$ & & $46,9 \mathrm{aB}$ & & $63,8 \mathrm{aA}$ \\
\hline \multicolumn{6}{|l|}{ Coeficiente de Variação (\%) 12,3 } \\
\hline \multicolumn{6}{|c|}{ Quociente Respiratório $\left(\mathrm{CO}_{2} \mathrm{O}_{2}^{-1}\right)$} \\
\hline (Teor de água nos níveis de secagem, \%) & $\mathrm{S}_{0}$ & $\mathrm{~S}_{\mathrm{I}}$ & & $\mathrm{S}_{\mathrm{II}}$ & Média \\
\hline $\operatorname{LAV}\left(\mathrm{S}_{0}=54 ; \mathrm{S}_{\mathrm{I}}=50 ; \mathrm{S}_{\mathrm{II}}=40\right)$ & 1,02 & 0,94 & & 0,99 & $0,99 \mathrm{a}$ \\
\hline $\operatorname{CAM}\left(\mathrm{S}_{0}=55 ; \mathrm{S}_{\mathrm{I}}=49 ; \mathrm{S}_{\mathrm{II}}=39\right)$ & 0,91 & 0,91 & & 0,88 & $0,90 \mathrm{a}$ \\
\hline $\mathrm{SBC}\left(\mathrm{S}_{0}=56 ; \mathrm{S}_{\mathrm{I}}=51 ; \mathrm{S}_{\mathrm{II}}=39\right)$ & 0,98 & 0,99 & & 0,98 & $0,98 \mathrm{a}$ \\
\hline $\operatorname{SBS}\left(\mathrm{S}_{0}=57 ; \mathrm{S}_{\mathrm{I}}=51 ; \mathrm{S}_{\mathrm{II}}=39\right)$ & 0,94 & 0,90 & & 1,14 & $0,99 \mathrm{a}$ \\
\hline $\operatorname{JUM}\left(\mathrm{S}_{0}=61 ; \mathrm{S}_{\mathrm{I}}=50 ; \mathrm{S}_{\mathrm{II}}=41\right)$ & 0,95 & 0,92 & & 0,99 & $0,95 \mathrm{a}$ \\
\hline $\operatorname{RIB}\left(\mathrm{S}_{0}=63 ; \mathrm{S}_{\mathrm{I}}=50 ; \mathrm{S}_{\mathrm{II}}=41\right)$ & 1,03 & 0,86 & & 0,87 & $0,92 \mathrm{a}$ \\
\hline $\operatorname{ITA}\left(\mathrm{S}_{0}=67 ; \mathrm{S}_{\mathrm{I}}=48 ; \mathrm{S}_{\mathrm{II}}=41\right)$ & 0,98 & 0,97 & & 1,01 & $0,98 \mathrm{a}$ \\
\hline Média & $0,97 \mathrm{~A}$ & $0,93 \mathrm{~A}$ & & $0,98 \mathrm{~A}$ & \\
\hline Coeficiente de Variação (\%) 9,17 & & & & & \\
\hline
\end{tabular}


Tabela 3. Consumo de oxigênio, produção de dióxido de carbono e quociente respiratório de sementes de Eugenia pyriformis Cambess. de distintas regiões de coleta, submetidas a diferentes temperaturas de incubação. Médias seguidas pela mesma letra (minúscula compara regiões de coleta e maiúscula compara temperaturas) não diferem entre si pelo teste de Tukey, a 5\%. \%. $\mathrm{S}_{0}$ : sem secagem; $\mathrm{S}_{\mathrm{I}}$ : primeiro nível de secagem; $\mathrm{S}_{\mathrm{II}}$ : segundo nível de secagem. LAV: Lavras; CAM: Campinas; SBC: São Bernardo do Campo; SBS: São Bento do Sapucaí; JUM: Jumirim; RIB: Ribeirão Preto; ITA: Itaberá.

Table 3. Oxygen consumption, carbon dioxide production and respiratory quotient of Eugenia pyriformis Cambess.seeds from different collection regions, submitted to different incubation temperatures. Means followed by the same letter (lower case compare collection regions and upper case compare temperatures) do not differ from each other by the Tukey test at $5 \%$. $\mathrm{S}_{0}$ : without drying; $\mathrm{S}_{\mathrm{I}}$ : first level of drying; $\mathrm{S}_{\mathrm{II}}$ : second level of drying. LAV: Lavras; CAM: Campinas; SBC: São Bernardo do Campo; SBS: São Bento do Sapucaí; JUM: Jumirim; RIB: Ribeirão Preto; ITA: Itaberá.

\begin{tabular}{lcccccc}
\hline \multirow{2}{*}{$\begin{array}{l}\text { Região de } \\
\text { coleta }\end{array}$} & 10 & 15 & 20 & 25 & 30 & 35 \\
\cline { 2 - 6 } & \multicolumn{5}{c}{ Oxigênio $\left(\mu \mathrm{mol}_{2} \mathrm{gMS}^{-1} \mathrm{~d}^{-1}\right)$} \\
LAV & $16,5 \mathrm{bC}$ & $18,5 \mathrm{bC}$ & $26,9 \mathrm{dBC}$ & $35,7 \mathrm{dABC}$ & $47,2 \mathrm{dAB}$ & $52,2 \mathrm{cA}$ \\
$\mathrm{CAM}$ & $27,1 \mathrm{bE}$ & $32,8 \mathrm{abDE}$ & $51,1 \mathrm{bcCD}$ & $60,9 \mathrm{cBC}$ & $75,1 \mathrm{cB}$ & $102,3 \mathrm{bA}$ \\
SBC & $33,1 \mathrm{abC}$ & $41,1 \mathrm{abC}$ & $68,5 \mathrm{abB}$ & $88,1 \mathrm{bB}$ & $121,4 \mathrm{bA}$ & $114,5 \mathrm{bA}$ \\
$\mathrm{SBS}$ & $23,4 \mathrm{bE}$ & $30,3 \mathrm{bDE}$ & $48,0 \mathrm{bcdCD}$ & $54,1 \mathrm{cdBC}$ & $71,7 \mathrm{cB}$ & $104,1 \mathrm{bA}$ \\
JUM & $14,4 \mathrm{bC}$ & $19,7 \mathrm{bBC}$ & $30,0 \mathrm{cdBC}$ & $41,9 \mathrm{cdB}$ & $41,9 \mathrm{~dB}$ & $71,9 \mathrm{cA}$ \\
RIB & $53,4 \mathrm{aE}$ & $54,9 \mathrm{aE}$ & $88,8 \mathrm{aD}$ & $120,8 \mathrm{aC}$ & $152,4 \mathrm{aB}$ & $178,3 \mathrm{aA}$ \\
ITA & $22,1 \mathrm{bD}$ & $22,4 \mathrm{abD}$ & $53,6 \mathrm{bcC}$ & $61,5 \mathrm{cBC}$ & $77,3 \mathrm{cB}$ & $107,7 \mathrm{bA}$
\end{tabular}

Coeficiente de Variação (\%) 15,7

Dióxido de Carbono $\left(\mu \mathrm{mol} \mathrm{CO} \mathrm{CMS}^{-1} \mathrm{~d}^{-1}\right)$

$\begin{array}{lcccccc}\text { LAV } & 20,3 \mathrm{aB} & 21,7 \mathrm{aB} & 31,1 \mathrm{cAB} & 41,6 \mathrm{cdAB} & 49,2 \mathrm{dA} & 53,6 \mathrm{dA} \\ \mathrm{CAM} & 33,7 \mathrm{aD} & 41,1 \mathrm{aCD} & 55,6 \mathrm{abBCD} & 60,9 \mathrm{bcBC} & 73,7 \mathrm{cB} & 98,6 \mathrm{bA} \\ \mathrm{SBC} & 30,8 \mathrm{aC} & 35,5 \mathrm{aC} & 63,1 \mathrm{aB} & 75,0 \mathrm{abB} & 101,0 \mathrm{abA} & 110,4 \mathrm{bA} \\ \mathrm{SBS} & 20,5 \mathrm{aD} & 29,1 \mathrm{aCD} & 46,4 \mathrm{abcBCD} & 49,5 \mathrm{cdBC} & 64,2 \mathrm{cdB} & 92,1 \mathrm{bcA} \\ \text { JUM } & 20,2 \mathrm{aB} & 23,3 \mathrm{aB} & 33,6 \mathrm{bcB} & 32,9 \mathrm{~dB} & 42,2 \mathrm{~dB} & 70,0 \mathrm{cdA} \\ \text { RIB } & 31,4 \mathrm{aC} & 39,1 \mathrm{aC} & 51,5 \mathrm{abcC} & 89,2 \mathrm{aB} & 119,7 \mathrm{aA} & 137,7 \mathrm{aA} \\ \text { ITA } & 28,3 \mathrm{aC} & 31,8 \mathrm{aC} & 61,3 \mathrm{aB} & 58,5 \mathrm{bcB} & 79,6 \mathrm{bcB} & 106,4 \mathrm{bA}\end{array}$

Coeficiente de Variação (\%) 17,2

$\begin{array}{lcccccc}\text { LAV } & 1,3 \mathrm{abA} & 1,2 \mathrm{bAB} & 1,2 \mathrm{aAB} & 1,2 \mathrm{aAB} & 1,0 \mathrm{aB} & 1,0 \mathrm{aB} \\ \mathrm{CAM} & 1,2 \mathrm{bAB} & 1,3 \mathrm{abA} & 1,1 \mathrm{abBC} & 1,0 \mathrm{abC} & 1,0 \mathrm{abC} & 1,0 \mathrm{aC} \\ \text { SBC } & 0,9 \mathrm{cA} & 0,7 \mathrm{cdA} & 0,9 \mathrm{cA} & 0,8 \mathrm{cdA} & 0,8 \mathrm{bcA} & 1,0 \mathrm{aA} \\ \mathrm{SBS} & 1,0 \mathrm{cA} & 0,9 \mathrm{cA} & 0,9 \mathrm{bcA} & 0,9 \mathrm{bcA} & 0,9 \mathrm{abcA} & 0,9 \mathrm{abA} \\ \text { JUM } & 1,4 \mathrm{aA} & 1,2 \mathrm{bB} & 1,1 \mathrm{abBC} & 0,8 \mathrm{cdD} & 1,0 \mathrm{aC} & 1,0 \mathrm{aC} \\ \text { RIB } & 0,6 \mathrm{dBC} & 0,7 \mathrm{dABC} & 0,6 \mathrm{dC} & 0,7 \mathrm{dAB} & 0,8 \mathrm{cA} & 0,8 \mathrm{bA} \\ \text { ITA } & 1,3 \mathrm{abAB} & 1,4 \mathrm{aA} & 1,2 \mathrm{aBC} & 0,9 \mathrm{bcD} & 1,0 \mathrm{aCD} & 1,0 \mathrm{aCD}\end{array}$

Coeficiente de Variação (\%) 6,7 
resultando em ativação de vias fermentativas da respiração (Dahal et al. 1996, Buckeridge et al. 2004). Por outro lado, em temperaturas acima de $30{ }^{\circ} \mathrm{C}$ ocorre aumento da taxa de respiração, porém, pode estar associado a processos de deterioração que ocorrem sob elevadas temperaturas, com consequências na desnaturação de enzimas (Marcos Filho 2005).

De acordo com as diferenças significativas obtidas entre os valores de consumo de oxigênio ou de produção de dióxido de carbono, as procedências dos materiais podem ser agrupadas em três categorias de intensidades respiratórias: categoria I, maior intensidade, sementes oriundas de RIB e ITA; categoria II, intensidade intermediária, sementes oriundas de JUM, SBC, CAM e SBS; categoria III, intensidade menor, sementes oriundas de LAV (tabela 2). Curiosamente, a separação poderia ser adotada, também, para o teor de água na dispersão: categoria I, as mais úmidas (63\%-67\% de base úmida), categoria II, as intermediárias (55\% - 61\% de base úmida) e categoria III, as mais secas ( $54 \%$ de base úmida). Ressalta-se mais uma vez que o estádio de maturação das sementes chamadas recalcitrantes as caracteriza como imaturas, e que o grau de recalcitrância está diretamente relacionado ao grau de maturidade no momento em que as sementes se desligam da planta-mãe (Barbedo et al. 2013).

Alguns resultados permitem enfatizar ainda mais essa relação (tabelas 1-3). Primeiramente, quando se comparam LAV e CAM, hipoteticamente as mais maduras dentre todas: quase todos os valores da tabela 1 são semelhantes, mas a massa seca das sementes de LAV foi maior, provavelmente por terem maior tempo de acúmulo de reservas, permitindo maturação mais avançada e, consequentemente, levando a maiores valores de germinação. Neste caso, o índice de chuvas em LAV deve ter favorecido o acúmulo de massa seca, uma vez que essas sementes acumularam menor quantidade de graus-dia. Em seguida, teríamos as hipoteticamente mais imaturas, RIB e ITA: as sementes dessas duas regiões tiveram o menor ciclo e foram dispersas com o maior teor de água. Suas taxas de respiração estiveram muito acima das demais em quaisquer condições. E, finalmente, as sementes das demais regiões, em posições intermediárias para todas as características, mas com uma curiosa comparação entre JUM e SBC, em que a primeira teve germinação bem mais elevada e, a $35^{\circ} \mathrm{C}$, respiração bem abaixo de $\mathrm{SBC}$. Considerando-se que quanto mais avançado no processo de maturação está a semente, maior o índice de desligamento do metabolismo (Hell et al. 2019), há evidente variação no grau de maturação em que as sementes de uma mesma espécie, Eugenia pyrifomris, são dispersas, conforme as condições ambientais.

Assim, a análise comparativa entre os dados ambientais, ou seja, graus-dia, chuva acumulada, temperatura máxima e mínima do ar e extrato do balanço hídrico sequencial (tabela 1 e figuras 1-2), durante a formação das sementes, e o teor de água inicial (sementes sem secagem) (tabela 1) e as taxas respiratórias (tabelas 2-3), permitiram observar que as sementes com valores iniciais maiores de teor de água foram formadas em ciclo de maturação menor e com menor quantidade de chuva acumulada (tabela 1 e figuras 1-2). Outro resultado interessante foi o conteúdo de massa seca. Por exemplo, sementes procedentes de LAV e SBS, as quais apresentaram menores valores de teor de água inicial (sementes sem secagem) e taxas respiratórias, apresentaram maiores valores de massa seca (tabelas 1-2). Sob tal contexto, os resultados sugerem prováveis diferenças na maturidade das sementes quando oriundas de diferentes regiões, podendo ter relações com as condições ambientais.

Ao longo dos últimos anos, muitos foram os avanços científicos perante as sementes sensíveis à dessecação, ou seja, a classificação das sementes em dois grandes grupos, as ortodoxas e recalcitrantes, sendo respectivamente, tolerantes e intolerantes à dessecação (Roberts 1973). O entendimento da tolerância à dessecação e a característica recalcitrante das sementes foi proposto como um processo contínuo e não como uma situação absoluta, no qual há gradientes de tolerância à dessecação entre as espécies, formado pelo máximo de ortodoxia em um extremo e o máximo de recalcitrância no outro (Walters 2000, Berjak \& Pammenter 2007). Mais recentemente, há a consideração de que as sementes recalcitrantes, sensíveis à dessecação, podem ser na verdade, sementes dispersas ainda imaturas (Barbedo et al. 2013).

A variabilidade na sensibilidade à dessecação e respostas germinativas às temperaturas de incubação estão atreladas às características genéticas das populações e também as condições ambientais durante o período de formação das sementes (Daws et al. 2004, Daws et al. 2006). Sob tal exposição, é muito provável que as variações nas taxas respiratórias entre as diferentes procedências de coleta de Eugenia pyriformis estariam associadas às condições ambientais e à maturidade das sementes. Possivelmente as sementes que foram formadas em condições ambientais mais favoráveis, avançaram mais em sua maturação, visto pelo teor de água e conteúdo de massa seca e, com isso, adquiriram características fisiológicas e bioquímicas que permitiram maior exposição à secagem e às temperaturas adversas à germinação.

As condições ambientais e a origem do material podem estar associadas às variações na tolerância à dessecação e nas respostas germinativas e, também, como enaltecido aqui, nas taxas respiratórias. Para Wilson et al. (1970) e Berjak \& Pammenter (2007), a atividade metabólica após a dispersão pode diferir em função do padrão de desenvolvimento das sementes. Condições do ambiente durante a formação influenciam no amadurecimento e nas respostas fisiológicas e metabólicas de sementes (Wilson et al. 1970, Daws et al. 2004, Daws et al. 2006). Durante a fase de acúmulo de reserva da maturação há uma grande demanda no consumo de oxigênio que diminui à medida que se aproxima da fase de dessecação, devido a formações 

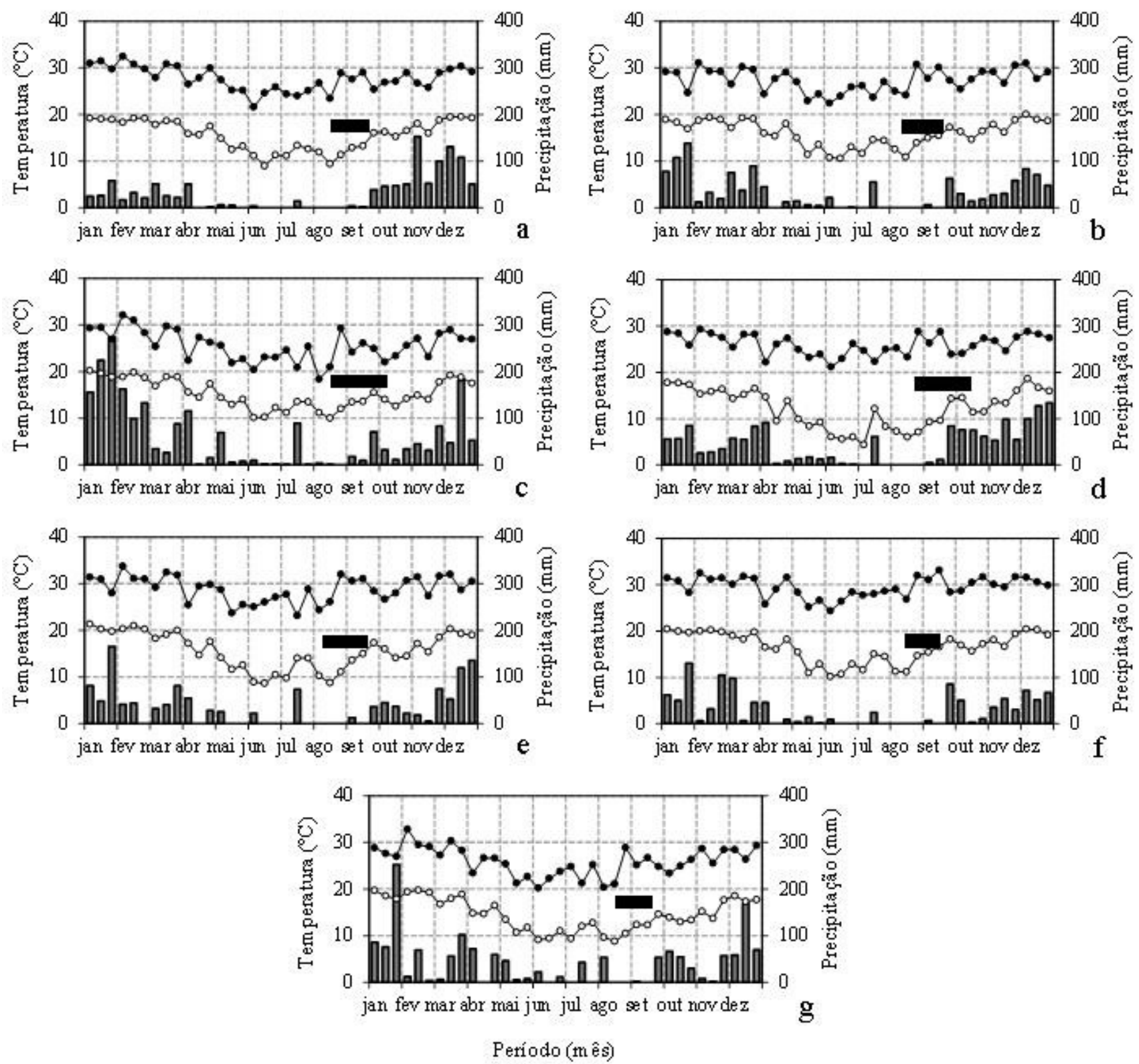

Figura 1. Dados meteorológicos de diferentes regiões de coleta de sementes de Eugenia pyriformis Cambess. a: Lavras - LAV; b: Campinas - CAM; c: São Bernardo do Campo - SBC; d: São Bento do Sapucaí - SBS; e: Jumirim - JUM; f: Ribeirão Preto - RIB; g: Itaberá - ITA. Temperatura máxima (círculos pretos), temperatura mínima (círculos brancos), precipitação pluvial (colunas cinza), período compreendido entre o florescimento e dispersão de sementes (barras pretas).

Figure 1. Meteorological data from different regions of Eugenia pyriformis Cambess. seed collection. a: Lavras - LAV; b: Campinas CAM; c: São Bernardo do Campo - SBC; d: São Bento do Sapucaí - SBS; e: Jumirim - JUM; f: Ribeirão Preto - RIB; g: Itaberá - ITA. Maximum temperature (black circles), minimum temperature (white circles), rainfall (gray columns), the period between flowering and seed dispersal (black bars).

de barreiras, como alterações anatômicas do tegumento e aumento da viscosidade celular, limitando a difusão do oxigênio e atenuando danos oxidativos nas membranas (Borisjuk \& Rolletschek 2009, Angelovici et al. 2010). Portanto, as variações nas taxas respiratórias para as sementes de Eugenia pyriformis podem estar associadas com o avanço da maturação na aquisição dessas e de outras características, uma vez que por serem sementes sensíveis à dessecação (Delgado \& Barbedo 2007), são dispersas antes da fase de dessecação da maturação (Barbedo et al. 2013).

Por fim, fez aqui uma análise da taxa respiratória de sementes de Eugenia pyriformis procedentes de diferentes 


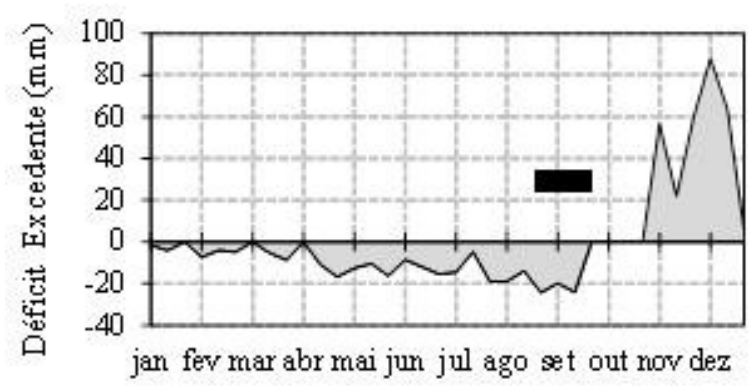

a
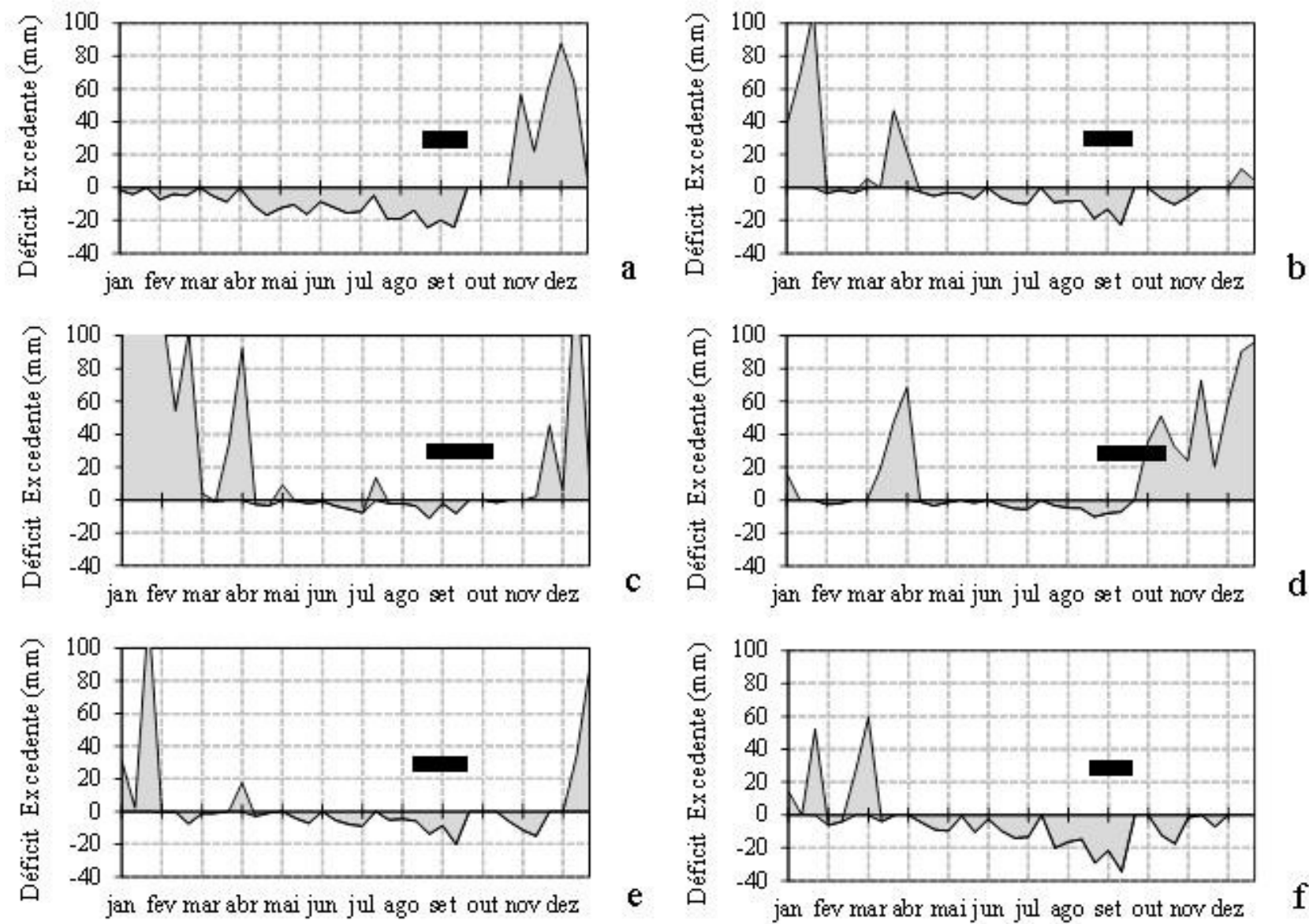

c
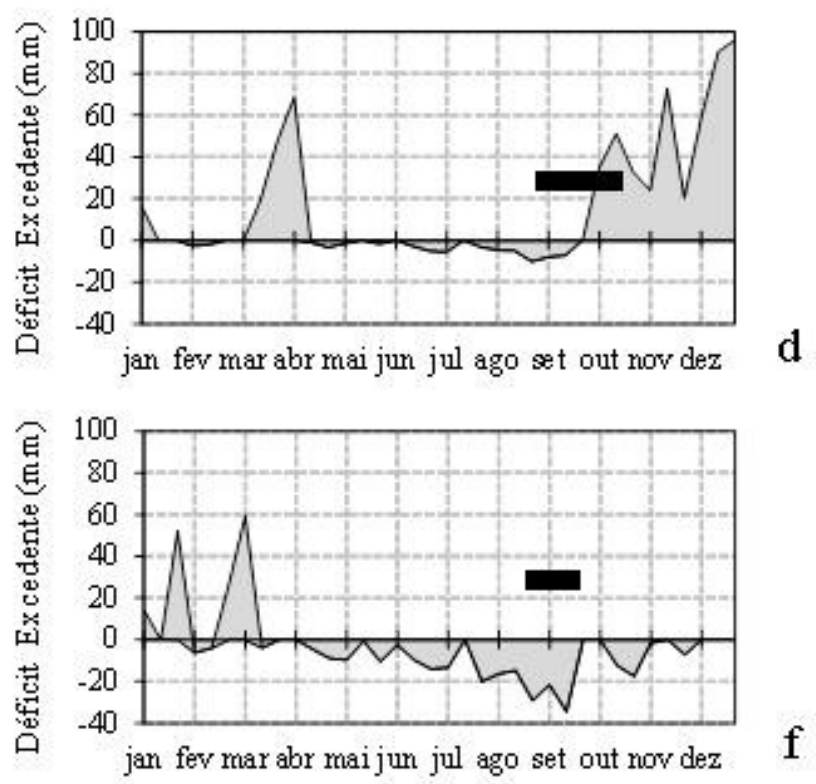

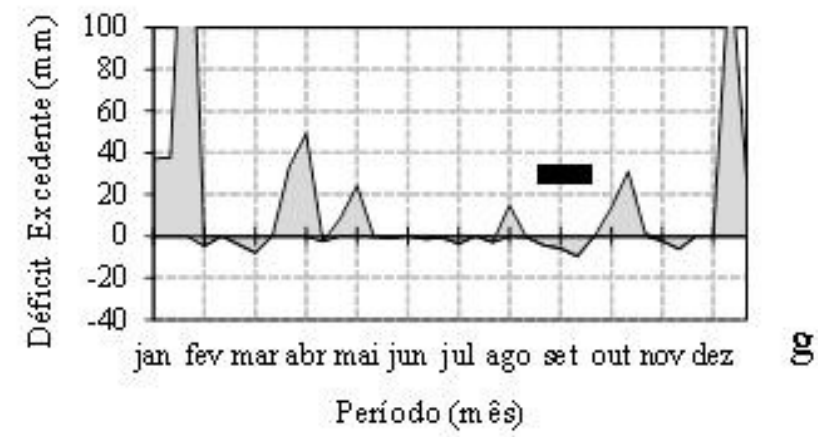

Figura 2. Extrato do balanço hídrico sequencial de diferentes regiões de coleta de sementes de Eugenia pyriformis Cambess. a: Lavras LAV; b: Campinas - CAM; c: São Bernardo do Campo - SBC; d: São Bento do Sapucaí - SBS; e: Jumirim - JUM; f: Ribeirão Preto - RIB; g: Itaberá - ITA. Período compreendido entre o florescimento e dispersão de sementes (barras pretas).

Figure 2. Sequential water balance extract from different regions of Eugenia pyriformis Cambess.seed collection. a: Lavras - LAV; b: Campinas - CAM; c: São Bernardo do Campo - SBC; d: São Bento do Sapucaí - SBS; e: Jumirim - JUM; f: Ribeirão Preto - RIB; g: Itaberá - ITA. Period between flowering and seed dispersal (black bars).

regiões e submetidas a diferentes níveis de secagem e diferentes temperaturas de incubação, incluindo-se, ainda, a análise das condições ambientais durante a formação das sementes. As sementes de Eugenia pyriformis apresentam variações na intensidade respiratória em função da origem do material, quando analisadas em diferentes níveis de secagem e em diferentes temperaturas de incubação. Tais variações nas taxas respiratórias podem estar associadas à maturidade das sementes, após a dispersão, visto, principalmente, pelo teor de água inicial, influenciadas pelas condições ambientais durante a formação das sementes. 


\section{Agradecimentos}

Os autores agradecem à Universidade Federal de Lavras (MG), ao Instituto Agronômico de Campinas, à USP de Ribeirão Preto (SP), à Casa da Agricultura em Jumirim (SP), à CATI de São Bento do Sapucaí (SP) e à CATI de Itaberá (SP), pela permissão e apoio nas coletas. Ao Instituto Agronômico de Campinas (SP), ao Instituto Astronômico e Geofísico da Universidade de São Paulo e à Universidade Federal de Lavras, pelo fornecimento dos dados meteorológicos. Ao Núcleo de Pesquisa em Sementes, Instituto de Botânica (SP), pela permissão e apoio na realização dos experimentos. À Dra. Lucia Rossi (Instituto de Botânica, SP), pela colaboração e identificação das espécies. Ao CNPq e CAPES, pela concessão de bolsas.

\section{Literatura citada}

Andrade, R.N.B. \& Ferreira, A.G. 2000. Germinação e armazenamento de sementes de uvaia (Eugenia pyriformis Camb.) - Myrtaceae. Revista Brasileira de Sementes 22: 118-125.

Angelovici, R., Galili, G., Fernie, A.R. \& Fait, A. 2010. Seed desiccation: a bridge between maturation and germination. Trends in Plant Science 15: 211-218.

Barbedo, C.J., Bilia, D.A.C. \& Figueiredo-Ribeiro, R.C.L. 2002. Tolerância à dessecação e armazenamento de sementes de Caesalpinia echinata Lam. (pau-brasil), espécie da Mata Atlântica. Revista Brasileira de Botânica 25: 431-439.

Barbedo, C.J., Centeno, D.C. \& Figueiredo-Ribeiro, R.C.L. 2013. Do recalcitrant seeds really exist?. Hoehnea 40: 583-593.

Berjak, P. \& Pammenter, N.W. 2007. From Avicennia to Zizania: seed recalcitrance in perspective. Annals of Botany 101: 213-228.

Bonjovani, M.R. \& Barbedo, C.J. 2019. Respiration and deterioration of Inga vera ssp. affinis embryos stored at different temperatures. Journal of Seed Science 41: 44-53.

Borghetti, F. \& Ferreira, A.G. 2004. Interpretação de resultados de germinação. In: A.G. Ferreira \& F. Borghetti (eds.). Germinação: do básico ao aplicado. Artmed, Porto Alegre. pp. 209-222.

Borisjuk, L. \& Rolletschek, H. 2009. The oxygen status of the developing seed. New Phytologist 182: 17-30.

Brasil. 2009. Regras para análises de sementes. Brasília, Ministério da Agricultura.

Buckeridge, M.S., Tiné, M.A.S., Minhoto, M.J. \& Lima, D.U. 2004. Respiração. In: G.K. Kerbauy, (ed.). Fisiologia vegetal. Guanabara Koogan, Rio de Janeiro, pp. 198-216.
Caccere, R., Teixeira, S.P., Centeno, D.C., FigueiredoRibeiro, R.C.L. \& Braga, M.R. 2013. Metabolica and structural changes during early maturation of Inga vera seeds are consistent with the lack of a desiccation phase. Journal of Plant Physiology 170: 791-800.

Dahal, P., Kim, N.S. \& Bradford, K.J. 1996. Respiration and germination rates of tomato seeds at suboptimal temperatures and reduced water potentials. Journal of Experimental Botany 47: 941-947.

Daws, M.I., Cleland, H., Chmielarz, P., Gorin, F., Leprince, O., Matthews, S., Mullins, C.E., Thanos, C.A., Vandvik, V. \& Pritchard, H.W. 2006. Variable dessication tolerance in Acer pseudoplatanus seeds in relation to developmental conditions: a case of phenotypic recalcitrance? Functional Plant Biology 33: 59-66.

Daws, M.I., Lydall, E., Chmielarz, P., Leprince, O., Matthews, S., Thanos, C.A. \& Pritchard, H.W. 2004. Developmental heat sum influences recalcitrant seed traits in Aesculus hippocastanum across Europe. New Phytologist 162: 157-166.

Delgado, L.F. \& Barbedo, C.J. 2007. Tolerância à dessecação de sementes de espécies de Eugenia. Pesquisa Agropecuária Brasileira 42: 265-272.

Hell, A.F., Kretzschmar, F.S., Simões, K., Heyer, A.G., Barbedo, C.J., Braga. M.R. \& Centeno, D.C. 2019. Metabolic changes on the acquisition of desiccation tolerance in seeds of the brazilian native tree Erythrina speciosa. Frontiers in Plant Science 10: article 1356.

Kader, A.A. \& Saltveit, M.E. 2002. Respiration and gas exchange. J.A. Bartz, J.K. Brecht. \& J, Weichmann. (eds.) In: Postharvest physiology and pathology of vegetables, Marcel Deckker: New York, pp.7-29.

Labouriau, L.G. 1983. A germinação das sementes. Organização dos Estados Americanos, Washington.

Lamarca, E.V. \& Barbedo, C.J. 2012. Short storability of Caesalpinia echinata seeds as a consequence of oxidative processes. Revista Hoehnea 39: 577-586.

Lamarca, E.V., Prataviera, J.S., Borges, I.F., Delgado, L.F., Teixeira, C.C., Camargo, M.B.P.de., Faria, J.M.R. \& Barbedo, C.J. 2013. Maturation of Eugenia pyriformis seeds under different hydric and thermal conditions. Anais da Academia Brasileira de Ciências 85: 223-233.

Lamarca, E.V.; Silva, C.V. \& Barbedo, C.J. 2011. Limites térmicos para a germinação em função da origem de sementes de espécies de Eugenia (Myrtaceae) nativas do Brasil. Acta Botanica Brasilica 25: 293-300.

Leprince, O., Buitink, J. \& Hoekstra, F.A. 1999. Axes and cotyledons of recalcitrant seeds of Castanea sativa Mill. exhibit contrasting responses of respiration to drying in relation to desiccation sensitivity. Journal of Experimental Botany 50: 1515-1524. 
Maguire, J. D. 1962. Speed of germination-aid in selection and evaluation for seedling emergence and vigor. Crop Science, Madison 2: 176-177.

Marcos Filho, J. 2005. Fisiologia de sementes de plantas cultivadas. FEALQ, Piracicaba.

Mattana, E., Daws, M.I., Fenu, G. \& Bacchetta, G. 2012. Adaptation to habitat in Aquilegia species endemic to Sardinia (Italy): Seed dispersal, germination and persistence in the soil. Plant Biosystems 146: 374-383.

Mayrinck, R.C., Vilela, L.C., Pereira, T.M., RodriguesJunior, A.G., Davide, A.C. \& Vaz, T.A.A. 2019. Seed desiccation tolerance/sensitivity of tree species from Brazilian biodiversity hotspots: considerations for conservation. Trees 33: 777-785. doi.org/10.1007/s00468-019-01815-8.

Pedro Junior, M.J., Brunini, O., Alfonsi, R.R. \& Angelocci, L.R. 1977. Estimativa de graus-dia em função de altitude e latitude para o estado de São Paulo. Bragantia 36: 89-92.

Roberts, E.H. 1973. Predicting the storage life of seeds. Seed Science and Technology 1: 499-514.

Rolim, G.S., Camargo, M.B.P.de., Lania, D.G. \& Moraes, J.F.L. 2007. Classificação climática de Köppen e de Thornthwaite e sua aplicabilidade na determinação de zonas agroclimáticas para o estado de São Paulo. Bragantia 66: 711-720.

Santana, D.G. \& Ranal, M.A. 2004. Análise da germinação: um enfoque estatístico. Universidade de Brasília, Brasília.

Scalon, S.P.Q., Neves, E.M.S., Maseto, T.E. \& Pereira, Z.V. 2012. Sensibilidade à dessecação e ao armazenamento em sementes de Eugenia pyriformis Cambess. (uvaia). Revista Brasileira de Fruticultura 34: 269-276.
Silva, E.A.A., Oliveira, J.M. \& Pereira, W.V.S. 2018. Fisiologia das sementes. In: Barbedo, C.J. \& Santos Junior, N.A. (orgs.). Sementes do Brasil: produção e tecnologia para espécies da flora brasileira. Instituto de Botânica, São Paulo. pp. 15-40.

Silva, L.A.da., Sales, J.de.F., Santo, H.O.dos., Martins, J.M., Costa, V.H. \& Silva, F.G. 2017. Physiological performance of cagaita seeds (Eugenia dysenterica DC.) subjected to drying. Semina: Ciências Agrárias 38: 19-34.

Stavrinides, A., Dussert. S., Combes, M.C., FockBastide, I., Severac, D., Minier, J., Bastos-Siqueira, A., Demolombe, V., Hem, S., Lashermes, P. \& Joët, T. 2019. Seed comparative genomics in three coffee species identify desiccation tolerance mechanisms in intermediate seeds. Journal of Experimental Botany erz508. doi:10.1093/jxb/erz508.

Thornthwaite, C.W. \& Mather, J.R. 1955. The water balance. New Jersey: Drexel Institute of Technology (Publications in Climatology).

Villa Nova, N.A., Pedro Júnior, M.J., Pereira, A.R. \& Ometto, J.C. 1972. Estimativa de graus-dia acumulados acima de qualquer temperatura base em função das temperaturas máxima e mínima. Caderno Ciência da Terra 30: 1-8.

Walters, C. 2000. Levels of recalcitrance in seeds. Revista Brasileira de Fisiologia Vegetal 12: 7-21.

Wilson, A. M. Nelson, J.R. \& Goebel, C.J. 1970. Effects of environment on the metabolism and germination of crested wheatgrass seeds. Journal of Range Management 23: $283-288$. 